Draft version September 26, 2018

Preprint typeset using IATEX style emulateapj v. 25/04/01

\title{
COLLISIONAL PARTICLE DISKS
}

\author{
Yoram Lithwick ${ }^{1}$ \& Eugene ChiAnG $^{2,3}$ \\ yoram@cita.utoronto.ca, echiang@astron.berkeley.edu \\ Draft version September 26, 2018
}

\begin{abstract}
We present a new, simple, fast algorithm to numerically evolve disks of inelastically colliding particles surrounding a central star. Our algorithm adds negligible computational cost to the fastest existing collisionless N-body codes, and can be used to simulate, for the first time, the interaction of planets with disks over many viscous times. Though the algorithm is implemented in two dimensions - i.e., the motions of bodies need only be tracked in a plane - it captures the behavior of fully three-dimensional disks in which collisions maintain inclinations that are comparable to random eccentricities. We subject the algorithm to a battery of tests for the case of an isolated, narrow, circular ring. Numerical simulations agree with analytic theory with regards to how particles' random velocities equilibrate; how the ring viscously spreads; and how energy dissipation, angular momentum transport, and material transport are connected. We derive and measure the critical value of the coefficient of restitution above which viscous stirring dominates inelastic damping and the particles' velocity dispersion runs away.
\end{abstract}

Subject headings: accretion disks, planets:rings

\section{INTRODUCTION}

How does a disk of collisional particles surrounding a star evolve in the presence of planets? The answer to this question has important implications. For example, after the planets of our Solar System accreted most of their mass, many small, rocky and icy bodies remained orbiting the Sun. Somehow, the planets eliminated most of these remnant planetesimals, while leaving some behind to form the asteroid belt, the Kuiper belt, and the Oort cloud. In the vicinity of Uranus and Neptune, the small bodies must have been highly collisional. Otherwise, these planets would have taken $10^{12}$ yr to form in situ (Goldreich et al. 2004). ${ }^{4}$ Yet virtually all simulations of the late stages of planet formation in the outer Solar System - such as those that model the migration of the ice giants, the resulting trapping of Kuiper belt objects into resonances, and the ejection of small bodies to the Oort cloud - neglect collisions. When the effects of collisions are accounted for, the current picture of the formation of planetary systems might change drastically.

Planetary rings provide another setting in which interparticle collisions play a crucial role. What are the origins of narrow rings shepherded by satellites? How do narrow rings settle into their special apse and node-aligned states (e.g., Chiang \& Culter 2004)? And how do rings backreact upon and shape the orbits of shepherd satellites? Our understanding of satellite-ring interactions bears on mysteries such as the origin of eccentricities of extra-solar giant planets (e.g., Goldreich \& Sari 2003).

Despite its importance, the behavior of particle disks in the presence of perturbing bodies is poorly understood. Numerical simulations can help to further understanding. But until now, simulations of collisional disks have been too inefficient to follow, say, how disks viscously spread in the long term. Collisions are traditionally simulated with a brute-force method (e.g., Brahic 1977; Wisdom \& Tremaine 1988): at each time step of the integration of the gravitational equations of motion, it is determined which pairs of particles might collide before the next timestep. These potential collision pairs are then integrated forwards in time with a much smaller timestep, to see if they really do collide. But this method is inefficient: a brute-force search for collision partners requires around $N_{\mathrm{tp}}^{2}$ operations at each timestep, where $N_{\text {tp }}$ is the number of test particles. In addition, most potentially colliding pairs do not collide, particularly in optically thin disks. Hence much computing time is wasted on missed collisions. More complex algorithms have been devised to reduce computing time (e.g., Lewis \& Stewart 2000; Charnoz et al. 2001). But these are still not nearly as fast as the fastest collisionless N-body codes, such as SWIFT (Levison \& Duncan 1994).

We sought a collision algorithm that (i) could be added to any N-body code, such as the freely-available SWIFT; (ii) contributes negligibly to the computational cost; (iii) is simple conceptually; (iv) is easy to code; and (v) follows correctly the long-term viscous evolution of disks in the presence of planets. We designed our algorithm to simulate a vertically optically thin disk of identical, collisional, massless, inelastic but indestructible test particles that feel the gravity of the Sun and of multiple planets. Complications that we do not include, such as the self-gravity of the particles, order-unity optical depths, and particles with differing sizes, spins, and cohesive strengths, could all affect the viscous evolution in ways that are not currently understood. But at this stage it seems wisest to ignore these complications, even though the algorithm could be modified to handle them. Viewed in its most basic terms,

${ }^{1}$ Canadian Institute for Theoretical Astrophysics, 60 St. George Street, Toronto ON M5S 3H8, Canada

2 Astronomy Department, University of California at Berkeley, Berkeley CA 94720, USA

3 Alfred P. Sloan Research Fellow

${ }^{4}$ In the terrestrial zone, the small bodies were also likely collisional, although the case is not as convincing there as it is in the outer Solar System (Goldreich et al. 2004). 
inelastic collisions dampen random velocities and act as a source of friction between neighboring streamlines. As long as our algorithm preserves this behavior, while conserving angular momentum and accounting for the loss of energy in inelastic collisions, it seems likely that it will properly model the long-term evolution of collisional disks. In the present paper, we test this assertion thoroughly when there are no planets, comparing in detail the results of our simulations with those of analytic theory. In a future paper, we shall test our algorithm in the presence of planets.

\section{THE COLLISION ALGORITHM}

The gravitational equations of motion of the Sun, planets, and massless test particles are integrated with the Wisdom-Holman mapping method (Wisdom \& Holman 1991), using the SWIFT subroutine package (Levison \& Duncan 1994).

We supplement SWIFT with a subroutine that simulates collisions between test particles in a disk with vertical optical depth

$$
\tau \sim N_{\mathrm{tp}} \frac{s^{2}}{\bar{r} \cdot \Delta}<1,
$$

where $N_{\text {tp }}$ is the number of test particles, $s$ is their size, and $\bar{r}$ and $\Delta$ are, respectively, the mean orbital radius and the radial width of the annulus that the particles occupy. In collisional particle disks, collisions tend to isotropize the velocity distribution. ${ }^{5}$ The collision time is $t_{\text {col }} \sim 1 /\left(n_{\mathrm{v}} s^{2} u\right)$, where $u$ is the 1 -D random speed and $n_{\mathrm{v}}$ is the volumetric number density, which is related to $\tau$ via $\tau \sim n_{\mathrm{v}} s^{2} u t_{\mathrm{orb}}$. Therefore

$$
t_{\mathrm{col}} \sim \frac{t_{\mathrm{orb}}}{\tau}
$$

The collision time is longer than the orbital time by the $u$-independent factor $1 / \tau$.

We capture this behavior with two-dimensional simulations in which all bodies have zero inclination. Every time step $d t$, a two-dimensional square grid is built, with each grid element having dimensions $s_{\text {grid }} \times s_{\text {grid }} ; s_{\text {grid }}$ can be thought of as the size of a particle. If two test particles fall in the same grid cell, and if their relative speed is negative (i.e., if they are approaching each other), then they collide with each other with probability $P_{\text {col }}=d t / t_{\text {orb }} \ll 1$, where $t_{\text {orb }}$ is the orbital time at the collision point. A random number generator is used to determine whether or not they actually collide.

To see that this algorithm gives the same collision time as Equation (2) (where $\tau$ is given by Eq. [1] with $s \rightarrow s_{\text {grid }}$ ), it is instructive to consider first a simpler algorithm that also yields the correct collision time. In this simpler algorithm, one waits for a time interval of $t_{\text {orb }}$ (instead of $d t$ ) before finding which particles fall in the same grid cell. Then two particles which do fall in the same grid cell, and have converging velocities, collide with probability $P_{\text {col }}=1$. Since the probability that a given particle lies in a cell occupied by a second particle is $\tau$, the collision time is $t_{\text {orb }} / \tau$, as required. ${ }^{6}$ Turning now to the algorithm that we actually use, since we apply this algorithm every time interval $d t$ (and not $t_{\text {orb }}$ ), we must correspondingly reduce the probability of a collision by $d t / t_{\text {orb }}$ in order to maintain the collision time at the value given by Equation (2).

Although carried out in only two dimensions, we emphasize that our algorithm models three-dimensional disks in which collisions maintain inclinations that are comparable to the random eccentricities. A truly two-dimensional disk is not realistic because collisions invariably generate outof-plane velocities. But if one could somehow prevent the generation of out-of-plane velocities, the collision time in such a disk would be $\sim s /(u \tau)$, which differs from Equation $(2)$ by the factor $s /\left(u t_{\text {orb }}\right)$. Since our algorithm satisfies Equation (2), it does not model truly two-dimensional disks.

As will be shown below, collisions drive the random speed of the particles to $u \gtrsim s_{\text {grid }} / t_{\text {orb }}$. Hence if two particles fall in the same grid cell at one time, they will usually fall in separate grid cells after one orbital period. Since collisions can potentially occur every time step, it might be thought that the same two particles can collide many times in succession - a behavior that we consider undesirable. But this behavior is avoided by the requirement that particles must be approaching each other for a collision to occur; immediately after they collide, their relative velocity reverses sign, and they are no longer candidates for a collision pair.

One of the main advantages of our algorithm is that the timestep is not restricted by the Courant condition. In a brute-force algorithm, one must restrict $d t \ll s / u$ in order to ensure that any two particles that fall within a distance $s$ of each other collide. This restriction on $d t$ can be very cumbersome when $u \gg s / t_{\text {orb }}$, as it will be whenever planets stir up the eccentricities. We avoid the Courant condition by treating the vertical dimension statistically: when two particles fall within the same two-dimensional grid cell, they need only collide a small fraction of the time because their vertical positions will, in general, differ. With our algorithm, we may choose $d t$ to be as large as is allowed by SWIFT, which is typically a significant fraction of the orbital time.

If two particles have been selected for a collision, i.e., if they lie in the same grid cell, are approaching each other, and are selected by the random number generator, then their velocities are updated as though the bodies were frictionless spheres whose surfaces touch (e.g., Trulsen 1971): the component of the relative velocity vector that lies parallel to the axis connecting the two particles is reversed in sign (from a converging velocity to a diverging one), and multiplied by the coefficient of restitution $\epsilon$, i.e., in obvious notation,

$$
u_{\mathrm{rel}, \|}^{\prime}=-\epsilon u_{\mathrm{rel}, \|}
$$

Neither the perpendicular component of the relative velocity vector, nor the velocity of the center of mass of the two colliders, nor the positions of the colliders are changed by the collision. A collision does not alter the sum of the angular momenta of both colliding bodies; hence the colli-

${ }_{5}$ More precisely, in optically thin disks the r.m.s. azimuthal speed is twice the r.m.s. radial speed; the r.m.s. vertical speed is comparable.

6 Although this simpler algorithm yields the correct collision time, we did not use it because it introduces an artificial frequency into the problem, set by the time interval at which the algorithm is applied $\left(\sim t_{\text {orb }}\right)$. When we attempted this algorithm, we found that a gap was cleared in the disk of test particles where the orbital period was exactly equal to this interval. 
sion algorithm exactly conserves total angular momentum. Note that a collision between two particles separated by distance $d$ changes the velocities of the particles as though each was a smooth sphere with radius $d / 2$. Since $d$ changes from collision to collision, the particles' sizes are effectively changing; they are only approximately $s_{\text {grid }}$.

The algorithm has now been completely described, aside from how the code finds which pairs of particles lie in the same grid cell. To find colliding pairs, the code first determines in which grid cell each particle lies. A grid cell is labelled by two integers, representing its location along the $\mathrm{x}$ - and $\mathrm{y}$-axes. Second, the code sorts the grid cells that contain test particles with the heapsort algorithm (Press et al. 1992). The sorted occupied grid cells are then checked to see if the same grid cell is repeated for two different particles. The step that takes the most time in the entire collision algorithm is the heapsort, which requires $\sim N_{\text {tp }} \ln N_{\text {tp }}$ operations. However, in the runs presented in this paper, with $N_{\mathrm{tp}}=10^{4}$ particles, it was found that the collision algorithm contributed negligibly to the running time of SWIFT.

\section{SIMULATIONS OF NARROW CIRCULAR RINGS}

In the remainder of this paper, we investigate circular rings of particles, without any planets. Circular rings are understood quite well theoretically (e.g., Lynden-Bell \& Pringle 1974; Brahic 1977; Goldreich \& Tremaine 1978; Shukhman 1984; Petit \& Henon 1987). Our goal is not only to test the collision algorithm, but also to develop diagnostics that can be used for the much more complicated case when planets are present.

The parameters of the simulation include the coefficient of restitution $(\epsilon)$, the size of a grid element $\left(s_{\text {grid }}\right)$, the number of test particles $\left(N_{\mathrm{tp}}\right)$, and the initial orbital elements of the test particles. The central body's mass is $1 M_{\odot}$. We simulate narrow rings with mean radius $\bar{r}=1$ AU and radial width $\Delta \ll \bar{r}$, and choose $d t=0.18$ years for the integration timestep.

A particle ring has two characteristic timescales (Brahic 1977). The shorter one is $t_{\mathrm{col}}$, the time for a particle to collide. The longer one is $t_{\text {diff }}$, the time for particles to diffuse across the ring's width. Random velocities relax to their equilibrium distribution on timescale $t_{\text {col }}$, while the ring density evolves on timescale $t_{\text {diff }}$. We investigate in turn the evolution of random velocities and of density.

\section{RANDOM VELOCITY EVOLUTION}

\subsection{Theory}

Collisions can both excite random velocities by drawing energy from the background Keplerian shear ("viscous stirring"), and damp random velocities because of finite inelasticity. When the coefficient of restitution $\epsilon<\epsilon_{*}$, where $\epsilon_{*}$ is a critical value, a typical collision damps random velocities. But there is a limit to how cold the particles get. Particles on circular orbits collide with one another at the Keplerian shearing speed $\sim s \Omega$, where $s$ is the size of a particle and $\Omega$ is the orbital angular frequency. In our collision routine, this relative speed is $\sim s_{\text {grid }} \Omega$. Since a single such collision redirects the particles onto non-circular orbits, the random velocity cannot fall below $\sim s_{\text {grid }} \Omega$, and the rms (root-mean-squared) eccentricity always relaxes to

$$
e_{\mathrm{rms}} \sim \frac{s_{\text {grid }}}{r}
$$

in the $\epsilon \ll \epsilon_{*}$ limit, where $r$ is the local disk radius.

By contrast, when $\epsilon>\epsilon_{*}$, a typical collision excites random velocities: viscous stirring dominates inelastic damping, and the rms eccentricity runs away.

In optically thin disks composed of equal-size particles, $\epsilon_{*}$ is determined solely by the angular dependence of the differential collisional cross-section. For frictionless spheres, $\epsilon_{*}=0.63$ (Goldreich \& Tremaine 1978). For frictional spinning spheres, $\epsilon_{*}=0.92$ (Shukhman 1984). For our collisional cross-section, we derive $\epsilon_{*}=\sqrt{7} / 5=0.529$ (Eq. [69]).

\subsection{Simulations: Approach to Velocity Equilibrium}

Figure 1 shows the time evolution of the rms eccentricity,

$$
e_{\mathrm{rms}} \equiv\left\langle e^{2}\right\rangle^{1 / 2}
$$

in three simulations, where \langle\rangle averages over all test particles. In each simulation, $s_{\text {grid }}=10^{-3} \mathrm{AU}$ and $N_{\mathrm{tp}}=10^{4}$. Particles were initially on orbits evenly spaced in semimajor axis between $\bar{r}-\Delta_{0} / 2$ and $\bar{r}+\Delta_{0} / 2$, where $\bar{r}=1$ $\mathrm{AU}$ and $\Delta_{0}=0.08 \mathrm{AU}$ (top hat profile). Initial eccentricities were identical, and initial longitudes and pericentre longitudes were random.

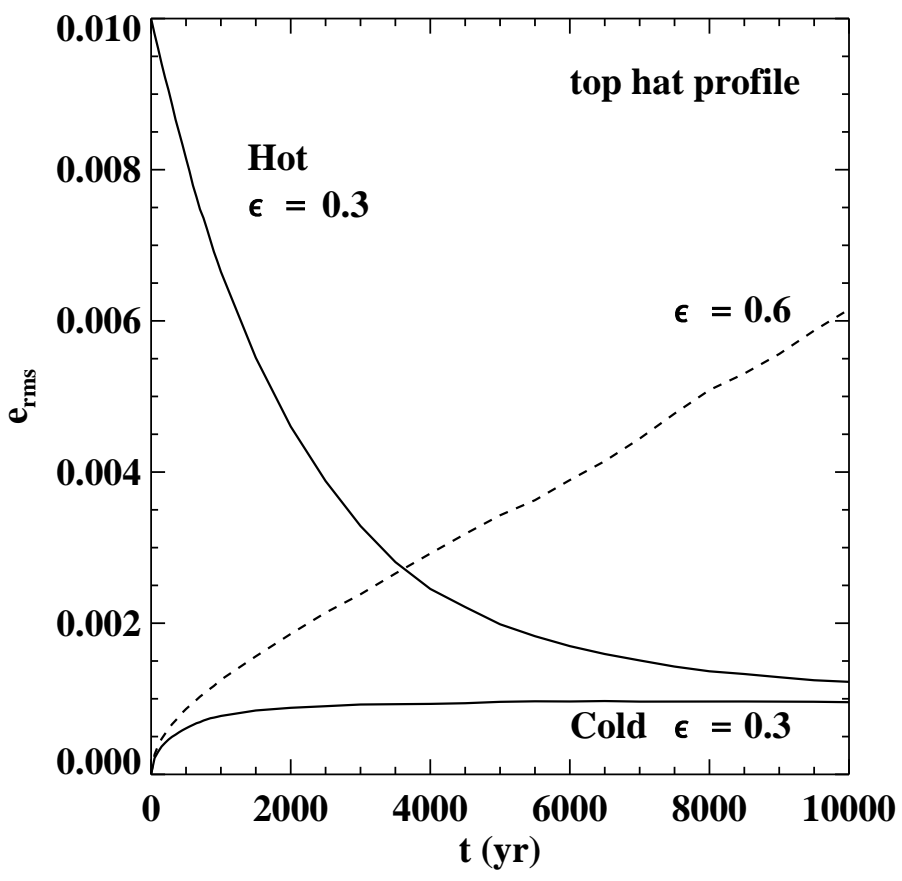

FIG. 1.- Random Velocity Evolution. The two simulations with $\epsilon=0.3$ both relax to the same $e_{\text {rms }} \sim s_{\text {grid }} / \bar{r}$. In the $\epsilon=0.6$ simulation, heating proceeds indefinitely.

Two simulations had $\epsilon=0.3$. One of these was initially cold, with initial eccentricities $=0$; the other was initially hot, with eccentricities $=0.01$. In both simulations, $e_{\mathrm{rms}}$ approached $\sim s_{\text {grid }} / \bar{r}=10^{-3}$ (Eq. [4]). Clearly, inelastic damping dominates viscous stirring when $\epsilon=0.3$. The third simulation had $\epsilon=0.6$, and was initially cold. Its $e_{\text {rms }}$ grew indefinitely. Hence viscous stirring dominates when $\epsilon=0.6$.

We define the collision time as

$$
t_{\mathrm{col}} \equiv \frac{N_{\mathrm{tp}} / 2}{\text { no. collisions per unit time }} \text {. }
$$


In the three simulations, we measured $t_{\mathrm{col}}=106-108$ yr. An estimator of $t_{\text {col }}$ from the input parameters of a simulation follows from a more precise form of Equations (1)-(2):

$$
t_{\text {col }}^{(\mathrm{est})}=t_{\mathrm{orb}} \frac{4 \pi r}{n s_{\text {grid }}^{2}},
$$

where

$$
n \equiv d N_{\mathrm{tp}} / d r
$$

is the number of particles per radial distance, and $4 \pi$ is the product of two factors: $2 \pi$ for the area of a ringlet $(2 \pi r \times d r)$, and 2 because only half of the time, when the relative velocity is negative, do doubly-occupied grid cells lead to collisions. For the parameters of the present simulations, with $r=\bar{r}$ and $d N_{\mathrm{tp}} / d r=N_{\mathrm{tp}} / \Delta_{0}, t_{\text {col }}^{(\text {est })}=101 \mathrm{yr}$. Because $t_{\text {col }}^{(\text {est })}$ does not account for the decreased collision frequency of particles at the edge of the ring, it underestimates $t_{\text {col }}$ by a small amount. That $t_{\text {col }}$ remained nearly constant throughout the simulation reflects the near constancy of the ring's width, since parameters were deliberately chosen to freeze out diffusion (from Eq. [9] below, the diffusion time in these simulations is $\sim 10^{5} \mathrm{yr}$ ).

In the cold $\epsilon=0.3$ simulation, $e_{\mathrm{rms}}$ reached 80 percent of its final value by time $t=1000 \mathrm{yr}$-around 10 collision times. The hot $\epsilon=0.3$ simulation took much longer to reach velocity equilibrium: initially, $e_{\mathrm{rms}}$ decayed approximately exponentially with a time constant of 2500 yr - around 25 collision times. Even after $t=2500 \mathrm{yr}$, the hot simulation took hundreds of collision times to reach its final $e_{\text {rms }}$. Velocity equilibration in the hot simulation was so long because of particles at ring edges. Edge particles tend to retain their initial eccentricities because their epicyclic excursions carry them away from the majority of particles; consequently, edge particles collide less frequently than do particles in the ring proper.

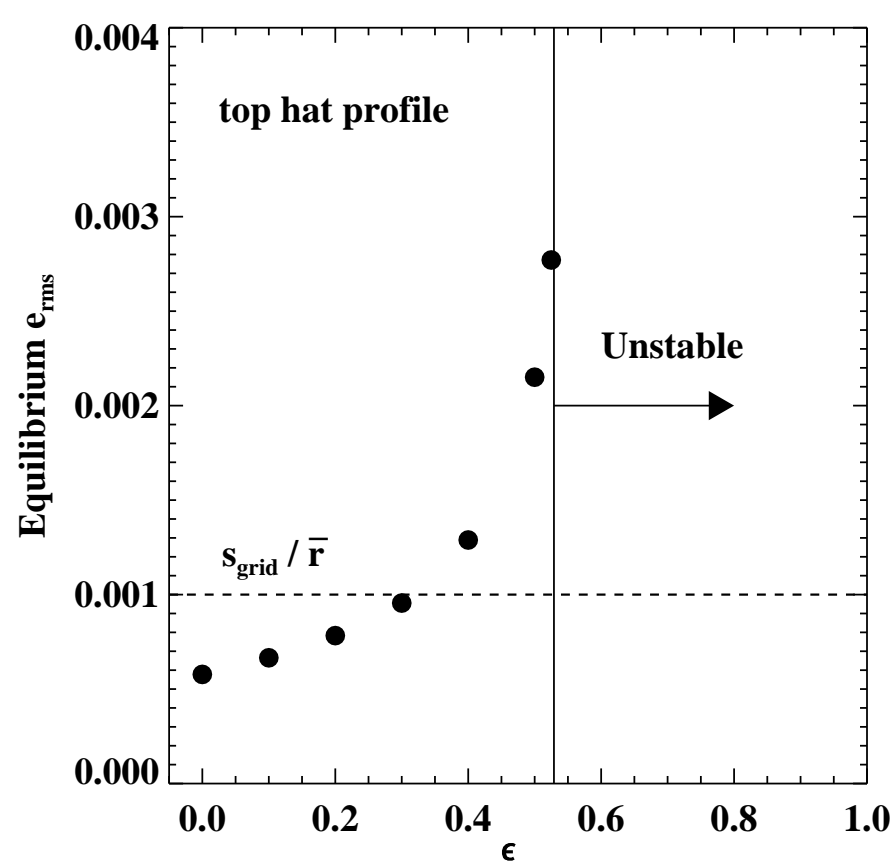

FIG. 2.- Equilibrium $e_{\mathrm{rms}}$. Seven points denote the steadystate $e_{\mathrm{rms}}$ in simulations with differing $\epsilon$. Vertical line shows critical value $\epsilon_{*}=\sqrt{7} / 5=0.529$. In initially cold simulations with $\epsilon=\{0,0.1,0.2,0.3\}, e_{\text {rms }}$ was evaluated at $10^{4} \mathrm{yr}$. In initially cold simulations with $\epsilon=\{0.4,0.5,0.525\}, e_{\text {rms }}$ was evaluated at $2 \times 10^{4}$ $\mathrm{yr}$, since these simulations took longer to reach steady state.

\subsection{Simulations: Equilibrium Eccentricities}

Figure 2 shows results from seven simulations, all with the same initial conditions as the cold simulations described in the previous subsection, except with differing values of $\epsilon$. The seven plotted simulations, with $\epsilon \leq 0.525$, all reached velocity equilibrium, with $e_{\mathrm{rms}} \sim$ (order unity constant) $\times s_{\text {grid }} / \bar{r}$. Simulations with $\epsilon \geq 0.6$ never reached velocity equilibrium. We conclude that $0.525<\epsilon_{*}<0.6$ for our collisional cross-section. In Equation (69) below, we derive $\epsilon_{*}=\sqrt{7} / 5=0.529$.

\section{DENSITY EVOLUTION}

\subsection{Theory}

A ring diffuses in the time that it takes a particle to random walk across its width. This random walk has a step-size equal to the epicyclic excursion of a particle $\left(\sim r e_{\mathrm{rms}} \sim s_{\text {grid }}\right)$ and a time per random step of $t_{\mathrm{col}}$. So to diffuse the width of the ring $\Delta$ takes a time

$$
t_{\text {diff }} \sim t_{\text {col }}\left(\frac{\Delta}{s_{\text {grid }}}\right)^{2} \gg t_{\text {col }},
$$

where the inequality holds when $\Delta \gg s_{\text {grid }}$; otherwise, $t_{\text {diff }} \sim t_{\text {col }}$ until $\Delta \sim s_{\text {grid }}$. Since $t_{\text {col }} \propto 1 / n \propto \Delta$ (Eq. [7]), a ring expands as

$$
\Delta \propto t^{1 / 3} .
$$

More precisely, $n$ satisfies the diffusion equation

$$
\frac{\partial n}{\partial t}=\frac{\partial}{\partial r}\left(\nu \frac{\partial n}{\partial r}\right),
$$

where the viscosity $\nu=$ constant $\times s_{\text {grid }}^{2} / t_{\text {col }}($ Eq. [9]). Inserting Equation (7) into our expression for $\nu$, we see 
that

$$
\nu=k_{\nu} \frac{s_{\text {grid }}^{4}}{\bar{r} t_{\text {orb }}} n,
$$

which defines the dimensionless constant $k_{\nu} ; k_{\nu}$ is a function of $\epsilon$, but is independent of $s_{\text {grid }}, \bar{r}$, and $n$. Petit \& Henon (1987) derived the above diffusion equation and gave its self-similar solution, an expanding inverted parabola:

$$
n=\frac{3}{2} \frac{N_{\mathrm{tp}}}{\Delta}\left(1-\left(\frac{r-\bar{r}}{\Delta / 2}\right)^{2}\right), \quad|r-\bar{r}| \leq \Delta / 2
$$

where

$$
\Delta \equiv\left(36 k_{\nu} \frac{s_{\text {grid }}^{4}}{\bar{r} t_{\text {orb }}} N_{\text {tp }} t\right)^{1 / 3} .
$$

Since the viscosity $\nu$ decreases with decreasing $n$, the diffusion is non-linear, and the edges of the ring at $r=\bar{r} \pm \Delta / 2$ are sharp.

\subsection{Simulations}

Because of the steep dependence of the diffusion timescale on the width of the ring, $t \propto \Delta^{3}$, it takes a long time to simulate even a modest increase in $\Delta$. Simulation parameters must be chosen judiciously. We fix $N_{\mathrm{tp}}=10^{4}$ and $\bar{r}=1 \mathrm{AU}$, and seek the optimal values for $s_{\text {grid }}$ and $\left.\Delta_{0} \equiv \Delta\right|_{t=0}$. To simulate as large an increase in $\Delta$ as possible, the simulation should begin with as narrow a ring as possible. For fixed $s_{\text {grid }}$, the narrowest ring that is not optically thick has unity optical depth: $\Delta_{0} \sim N_{\text {tp }}\left(s_{\text {grid }}\right)^{2} / 2 \pi \bar{r}$. The evolution timescale at the start of the simulation is $t_{0}=$ constant $\times\left(\Delta_{0}\right)^{3} /\left(s_{\text {grid }}\right)^{4}$ (Eq. [14]), so with unity optical depth, $t_{0}=$ constant $\times \Delta_{0}$. Hence the fastest timescale is obtained with the smallest $\Delta_{0}$. But we must have $\Delta_{0} \geq s_{\text {grid }}$, so the optimal values are $\Delta_{0}=s_{\text {grid }}=2 \pi \bar{r} / N_{\mathrm{tp}}$. Rounding up, we set $\Delta_{0}=s_{\text {grid }}=10^{-3}$ AU.

Figures 3 and 4 portray results from a simulation with the parameters listed above, and $\epsilon=0.3$. Initially, the ring particles were uniformly distributed in a ring with edges at $1 \pm\left(5 \times 10^{-4}\right)$ AU. In Figure 3, we show how the particles' dispersion in $r$,

$$
\sigma_{r} \equiv\left\langle(r-\bar{r})^{2}\right\rangle^{1 / 2},
$$

and how the collision time $\left(t_{\mathrm{col}}\right)$ vary with time, according to both theory and simulation. Figure 4 displays comparisons between theory and simulation for $n$. According to the theory described in $\S 5.1, n(r)$ is given by Equation (13), the dispersion in $r$ is

$$
\sigma_{r}^{2}=\int\left(n / N_{\mathrm{tp}}\right)(r-\bar{r})^{2} d r=\Delta^{2} / 20
$$

with $\Delta$ given by Equation (14), and the collision time is

$$
t_{\mathrm{col}}=\left(\int \frac{1}{N_{\mathrm{tp}}} \frac{n}{t_{\mathrm{col}}^{\text {(est) }}} d r\right)^{-1}=120 \pi k_{\nu} \frac{\left(s_{\text {grid }}\right)^{2}}{\Delta^{2}} t .
$$

The theory fits the numerical results well when we set

$$
k_{\nu}=0.016 .
$$

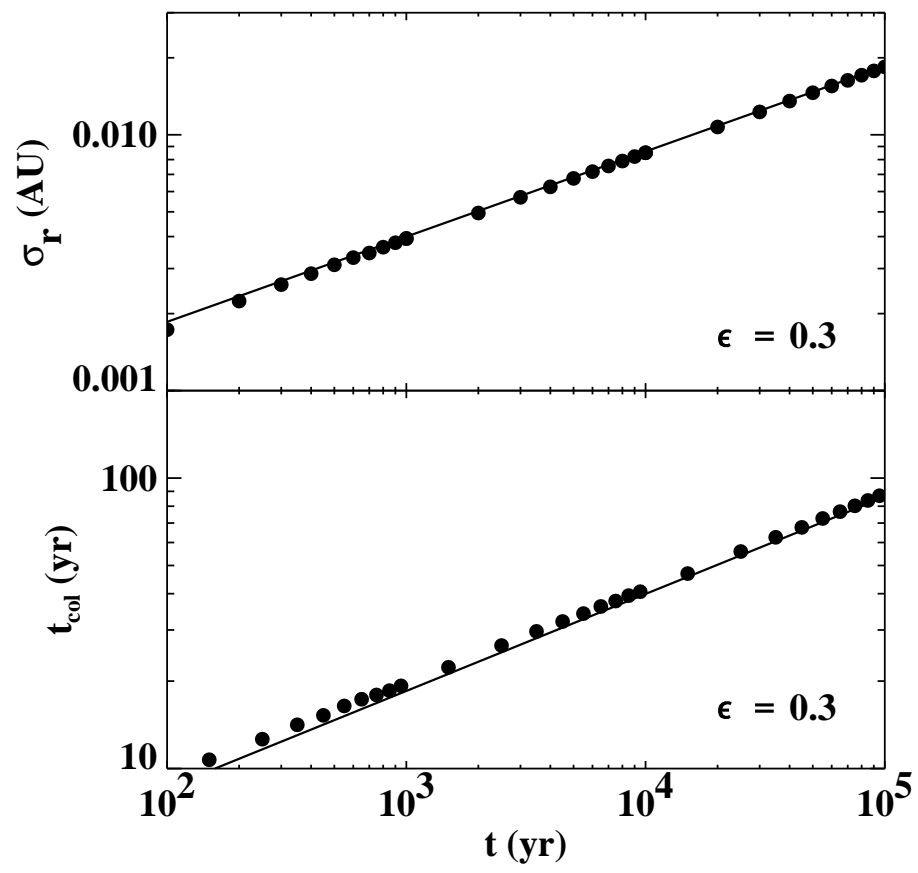

FIG. 3.- Dispersion and Collision Rate Evolution in a Diffusing Ring. Top panel: points show $\sigma_{r}=\left\langle(r-\bar{r})^{2}\right\rangle^{1 / 2}$ from the numerical simulation. The theory line through the points is $\Delta / \sqrt{20}$ (Eq. [16]), where $\Delta$ is given by Equation (14). The normalization of the line was adjusted by choosing $k_{\nu}=0.016$. Bottom panel: points show the collision time $t_{\mathrm{col}}$ from the simulation (Eq. [6]). The theory line is given by Equation (17), with $k_{\nu}=0.016$. Theory underestimates $t_{\mathrm{col}}$ by a small amount because of particles at ring edges $(\S 4.2)$.

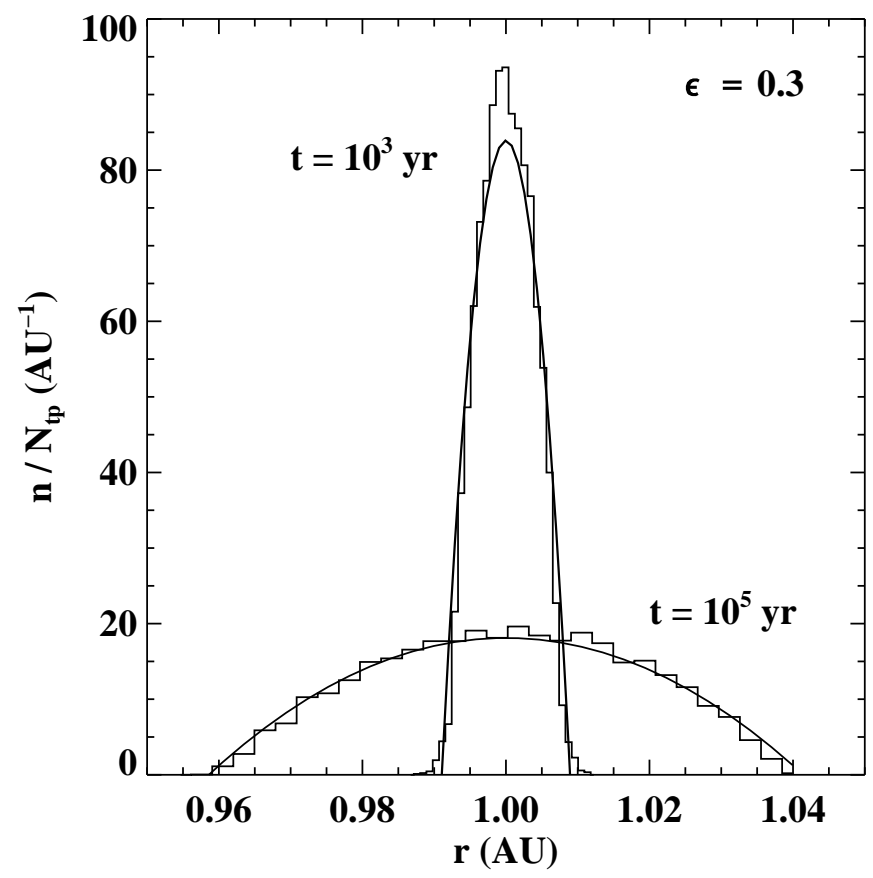

FIG. 4.- Density Evolution in a Diffusing Ring. Histograms of the number density are shown at two times from the same simulation as in Figure 3. The theory lines through the histograms are given by Equation (13), with $k_{\nu}$ the same as in Figure 3.

\section{ANGULAR MOMENTUM AND ENERGY TRANSPORT}

Understanding how planets interact with disks requires understanding how angular momentum and energy are transported - both within disks and between planets and disks. Below we study transport in isolated, circular, nar- 
row rings, developing diagnostics that will prove useful in future simulations of disks with planets.

\subsection{Theory}

The azimuthally-averaged equations describing the conservation of particle number, angular momentum, and energy are

$$
\begin{gathered}
\partial_{t} n+\partial_{r} F_{n}=0 \\
\partial_{t}(H n)+\partial_{r}\left(H F_{n}\right)=-\partial_{r} F_{H}^{\mathrm{visc}} \\
\partial_{t}(E n)+\partial_{r}\left(E F_{n}\right)=-\partial_{r} F_{E}^{\mathrm{visc}}-n \dot{\mathcal{E}},
\end{gathered}
$$

where $F_{n}$ is the (net) number flux across a circle of radius $r$, i.e., the number of particles per unit time that exit this circle minus the number that enter it; $H(r) \equiv\left(G M_{\odot} r\right)^{1 / 2}$ is the specific angular momentum of a particle on a circular orbit; $E(r) \equiv-G M_{\odot} / 2 r$ is the specific energy; $F_{H}^{\mathrm{visc}}$ (the "viscous flux of angular momentum") is defined as the difference between the total angular momentum flux and $H F_{n}$, i.e., $F_{H}^{\mathrm{visc}}+H F_{n}=F_{H}^{\mathrm{tot}} ; F_{E}^{\mathrm{visc}}$ is the corresponding difference in energy fluxes, i.e., $F_{E}^{\text {visc }}+E F_{n}=F_{E}^{\text {tot }}$; and $\dot{\mathcal{E}}$ is the rate at which specific energy is lost, per particle, in inelastic collisions.

Our decomposition of $F_{H}^{\text {tot }}$ into two components has the following interpretation: $H F_{n}$ is the angular momentum flux that would be carried by particles on circular orbits whose radii change on timescales long compared to the orbit time, while $F_{H}^{\text {visc }}$ is the part of the angular momentum flux not associated with the direct advection of circular orbits. The viscous flux of angular momentum and the corresponding viscous flux of energy are transferred in the ratio appropriate for circular orbits, i.e., since circular orbits have $E=-(1 / 2)\left(G M_{\odot} / H\right)^{2}$, a transfer in angular momentum of $\delta H$ must be accompanied by a transfer in energy of $\delta E=\left(G M_{\odot}\right)^{2} \delta H / H^{3}=\Omega \cdot \delta H$, so

$$
\frac{F_{E}^{\text {visc }}}{F_{H}^{\text {visc }}}=\Omega(r)
$$

The above relation applies only to the viscous fluxes, not to the total fluxes (i.e., $E F_{n} / H F_{n}=-\Omega / 2$ ). Equations (20) and (21) simplify with the aid of Equations (19) and (22) to

$$
\begin{aligned}
F_{n} & =-\frac{2}{r \Omega} \partial_{r} F_{H}^{\mathrm{visc}} \\
F_{H}^{\mathrm{visc}} & =-n \dot{\mathcal{E}} /(d \Omega / d r) .
\end{aligned}
$$

The latter is the well-known relation between energy dissipation and viscous angular momentum flux for accretion disks (e.g., Lynden-Bell \& Pringle 1974).

Since $\dot{\mathcal{E}} \sim s_{\text {grid }}^{2} \Omega^{2} / t_{\text {col }} \sim n s_{\text {grid }}^{4} \Omega^{2} / 4 \pi r t_{\text {orb }}$, we may rewrite (19) using (23) and (24) as

$$
\partial_{t} n=\left(\frac{k_{E} s_{\text {grid }}^{4}}{2 r t_{\text {orb }}}\right) \partial_{r}^{2} n^{2}
$$

where

$$
k_{E} \equiv \frac{n \dot{\mathcal{E}}}{n^{2} s_{\text {grid }}^{4}\left(3 \Omega^{2} / 8 r t_{\text {orb }}\right)}
$$

is an $\epsilon$-dependent dimensionless constant. In deriving (25), we have dropped terms that are small for narrow rings (e.g., $\left.\left.|d(\ln \Omega) / d r| \ll \mid d\left(\ln n^{2}\right) / d r\right) \mid\right)$. Equation (25) is identical to Equations (11)-(12) provided $k_{E}=k_{\nu}$.

\subsection{Simulations}

In this subsection, we diagnose angular momentum and energy transport in numerical simulations and compare to theory. To measure the number flux $F_{n}$ across a circle of radius $r$, we evaluate $N_{>r}$, the number of particles whose radial distances from the Sun exceed $r$, at two times, $t_{m}$ and $t_{m}+d t_{m}$. Then

$$
F_{n}\left(r, t_{m}\right) \doteq \frac{N_{>r}\left(t_{m}+d t_{m}\right)-N_{>r}\left(t_{m}\right)}{d t_{m}},
$$

where the symbol $\doteq$ means that this is how $F_{n}$ is measured. Similarly, we measure the total angular momentum flux as

$$
F_{H}^{\mathrm{tot}}\left(r, t_{m}\right) \doteq \frac{H_{>r}\left(t_{m}+d t_{m}\right)-H_{>r}\left(t_{m}\right)}{d t_{m}},
$$

where $H_{>r}$ is the sum of the specific angular momenta of all particles whose radial distances exceed $r$. For the energy flux, we must account for the energy lost in inelastic collisions (where the specific energy lost per collision $=u_{\mathrm{rel}, \|}^{2}\left(1-\epsilon^{2}\right) / 4$; see Eq. [3]). To this end, when calculating the energy flux across a circle with radius $r$, we first calculate $\delta \mathcal{E}_{>r}$, the total specific energy lost between times $t_{m}$ and $t_{m}+d t_{m}$ in all inelastic collisions that occurred at radii $>r$. Then the total energy flux is

$$
F_{E}^{\mathrm{tot}}\left(r, t_{m}\right) \doteq \frac{E_{>_{r}}\left(t_{m}+d t_{m}\right)-E_{>_{r}}\left(t_{m}\right)+\delta \mathcal{E}_{>r}}{d t_{m}}
$$

where $E_{>r}$ is the sum of the specific energies of particles with radii greater than $r$. The viscous fluxes are determined by the three fluxes above:

$$
\begin{aligned}
& F_{H}^{\mathrm{visc}} \doteq F_{H}^{\mathrm{tot}}-H F_{n} \\
& F_{E}^{\mathrm{visc}} \doteq F_{E}^{\mathrm{tot}}-E F_{n} .
\end{aligned}
$$

Energy dissipation in an annulus between radius $r_{1}$ and $r_{2}$ is measured via

$$
n \dot{\mathcal{E}} \doteq \frac{\delta \mathcal{E}_{>r_{1}}-\delta \mathcal{E}_{>r_{2}}}{\left(r_{2}-r_{1}\right) d t_{m}} .
$$

Figure 5 shows a number of measurements of the angular momentum flux for the simulation whose parameters are given in $§ 5.2$. The theory curve is from Equations (24) and (26):

$$
F_{H}^{\mathrm{visc}}=k_{E} \frac{n^{2} s_{\text {grid }}^{4} \Omega}{4 t_{\mathrm{orb}}},
$$

with $n$ given by Equation (13), and $k_{E}=k_{\nu}=0.016$ (we verify the equality of $k_{E}$ and $k_{\nu}$ in Figure 7 below). Overlaid on this theory curve are three sets of datapoints, measured in three independent ways. The agreement between theory and simulations is excellent.

Figure 6 shows the number fluxes for the same simulation. The theory line shows (Eqs. [23],[33])

$$
F_{n}=-k_{E} \frac{s_{\text {grid }}^{4}}{2 \bar{r} t_{\text {orb }}} \frac{\partial n^{2}}{\partial r}
$$

with $n$ from Equation (13). The datapoints agree well with the theory, although there is some scatter.

The circles in Figure 7 show the energy dissipation constant $k_{E}$ (Eq. [26]) for the simulations described in $\S \S 4.2-$ 4.3, at the same output times (Fig. 2). Recall that each of these simulations has a top hat density profile and is run for much less than a viscous time, so the density hardly 
evolves. The simulation with $\epsilon=0.3$ has $k_{E}=0.016$. Therefore $k_{E}=k_{\nu}$ (Eq. [18]), as suggested below Equation (26). Note that it is much more efficient to measure $k_{E}$ than $k_{\nu}$, since $k_{E}$ can be measured in only a few collision times, whereas $k_{\nu}$ must be measured on the viscous timescale. We defer to $\S 6.4$ a discussion of the diamonds and stars in Figure 7.

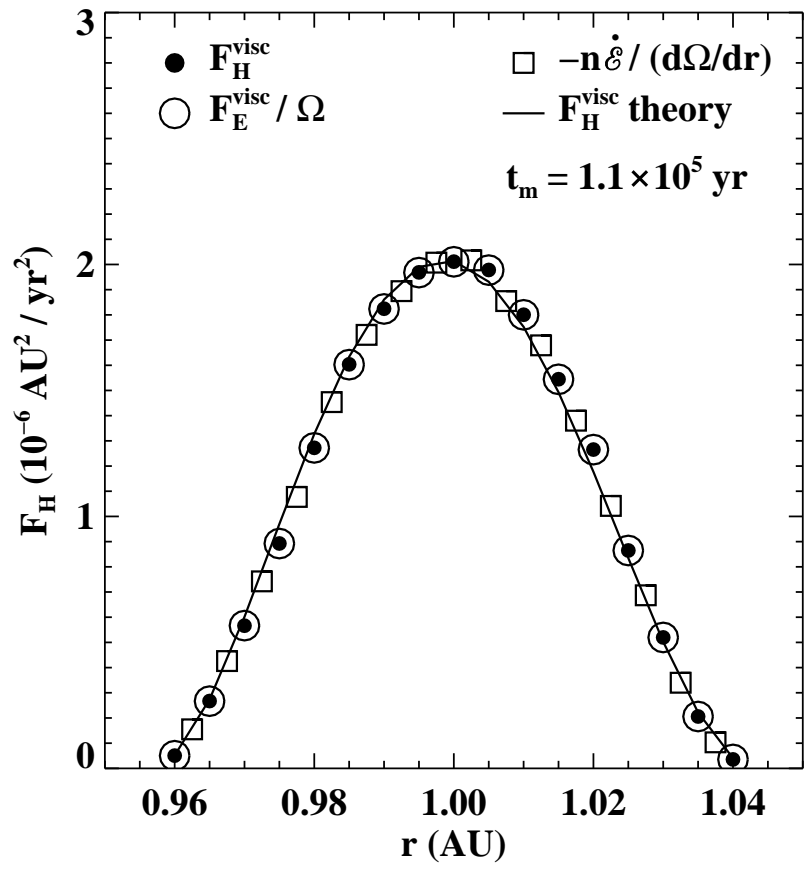

FIG. 5.- Angular Momentum Flux Measured With Various Methods. Data are taken from the simulation described in $\S 5.2$ (Figs. 3-4) at time $t_{m}=1.1 \times 10^{5} \mathrm{yr}$, with measurement interval $d t_{m}=10^{4}$ yr. The theory line is Equation (33). $F_{H}^{\mathrm{visc}}$ is measured with Equation (30). $F_{E}^{\text {visc }}$ is measured with Equation (31); clearly $F_{E}^{\text {visc }} / \Omega=F_{H}^{\text {visc }}$, confirming Equation $(22) . n \dot{\mathcal{E}}$ is measured with Equation (32); the squares confirm Equation (24).

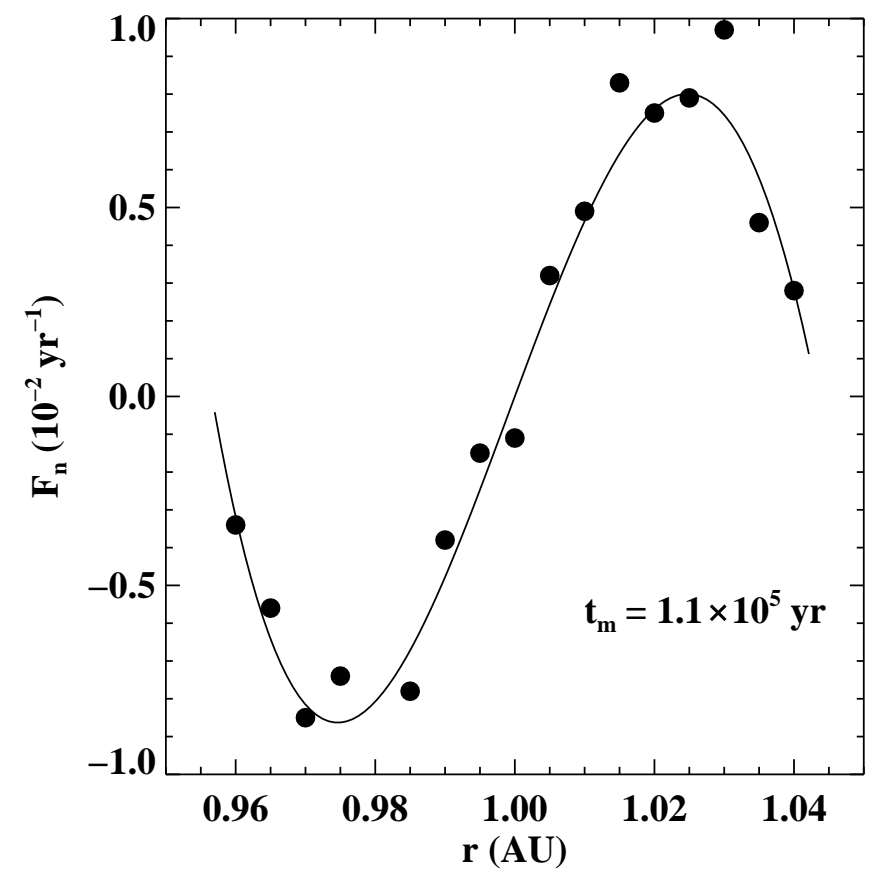

Fig. 6.- Number Flux. Data are taken from the same simulation as in Figure 5, and at same times. The theory line is Equation (34). Datapoints are measured with Equation (27).

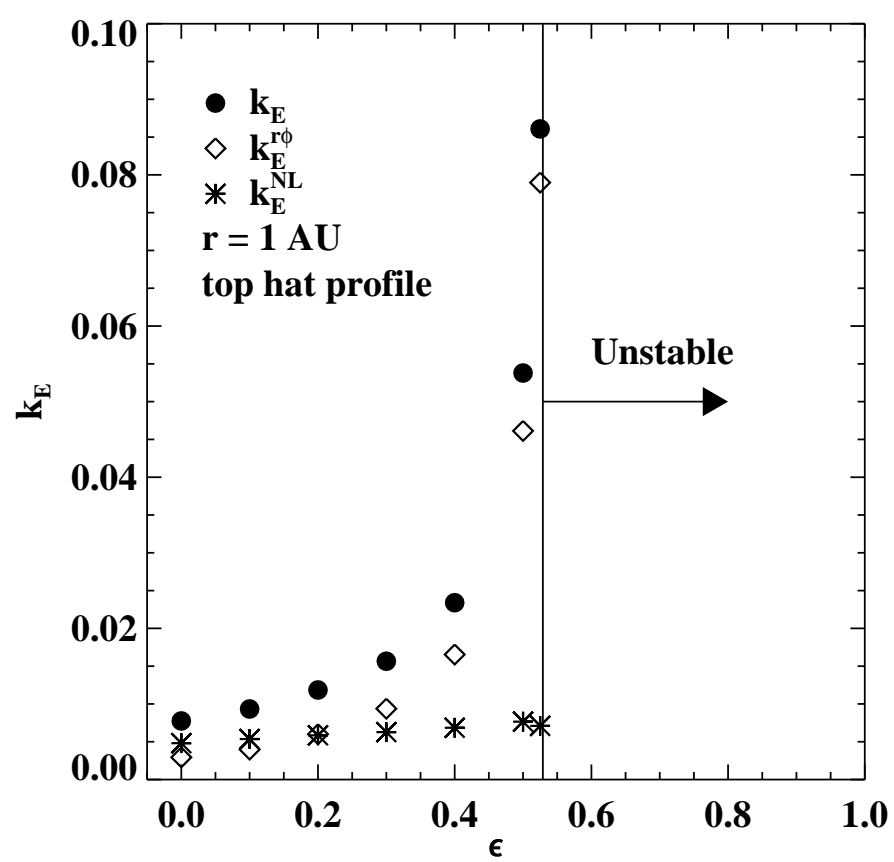

FIG. 7.- Energy Dissipation. The simulations are the same as those described in Figure 2. Circles show $k_{E}$, measured with Equations (26) and (32). Measurement parameters include $r_{1}=0.99$ $\mathrm{AU}, r_{2}=1.01 \mathrm{AU}, d t_{m}=5000 \mathrm{yr}$, and $t_{m}=5000 \mathrm{yr}$ for $\epsilon=\{0,0.1,0.2,0.3\}$ and $t_{m}=15000 \mathrm{yr}$ for $\epsilon=\{0.4,0.5,0.525\}$. For $\epsilon=0.3, k_{E}=0.016$, a value that matches $k_{\nu}$ as given by Equation (18). Diamonds show $k_{E}^{r \phi} \propto F_{r \phi}$, measured as described in $\S 6.4$. Stars give $k_{E}^{\mathrm{NL}}=k_{E}-k_{E}^{r \phi} \propto F_{\mathrm{NL}}$; this quantity is practically constant with $\epsilon$, as expected from Equation (52). Most of the energy dissipation (viscous transport of angular momentum) arises from $F_{r \phi}$ and not from $F_{\mathrm{NL}}$ as $\epsilon$ approaches $\epsilon_{*}$; compare with Equations (51)-(52).

\subsection{Dynamics from a Microscopic Perspective: Theory}

The angular momentum flux advected by particles is

$$
\begin{aligned}
& =n\left\langle r\left(\Omega r+v_{\phi}\right) v_{r}\right\rangle \\
& =H F_{n}+n r\left\langle v_{r} v_{\phi}\right\rangle,
\end{aligned}
$$

where $\boldsymbol{v}=\left(v_{r}, v_{\phi}\right)$ is the difference between a particle's total velocity (in the radial $r$ and azimuthal $\phi$ directions) and the Keplerian circular velocity at its position, and \langle\rangle denotes an average over particles in a narrow ring. Defining

$$
F_{r \phi} \equiv n r\left\langle v_{r} v_{\phi}\right\rangle
$$

we have

$$
F_{H}^{\mathrm{tot}}=H F_{n}+F_{r \phi}+F_{\mathrm{NL}},
$$

where $F_{\mathrm{NL}}$ is the "non-local" flux, i.e, the angular momentum flux not advected by particles (Wisdom \& Tremaine 1988). In $§ 6.1$, we considered only the combination

$$
F_{H}^{\mathrm{visc}}=F_{r \phi}+F_{\mathrm{NL}} \text {. }
$$

In this subsection, we wish to calculate $F_{H}^{\text {visc }}$ in terms of microscopic quantities. Hence we must consider the two components of $F_{H}^{\mathrm{visc}}$ separately. The two components have the following interpretation: (i) A particle that crosses a circle of radius $r$ has an angular momentum $H^{\prime}$ that is not, in general, equal to $H(r)$; the difference $H^{\prime}-H(r)$ contributes to $F_{r \phi}$. (ii) When a particle that is inside of the circle collides with one that is outside, the angular momentum transferred across the circle contributes to $F_{\mathrm{NL}}$. 
For a collisionless Keplerian particle, to lowest order in $e$,

$$
\begin{aligned}
& v_{r}=e r \Omega \sin (\Omega t) \\
& v_{\phi}=\frac{1}{2} e r \Omega \cos (\Omega t),
\end{aligned}
$$

with $r, \Omega$ independent of time. Therefore for collisionless particles, $F_{r \phi} \propto\left\langle v_{r} v_{\phi}\right\rangle \propto\langle\sin (2 \Omega t)\rangle=0$. But collisions give a definite contribution to $\left\langle v_{r} v_{\phi}\right\rangle$ (Greenberg 1988), as we presently show.

Instead of averaging over space only (as denoted by $\left\langle v_{r} v_{\phi}\right\rangle$ ), it will prove convenient to average over both space and time. We average over a radial width $\Delta r$ that is larger than the particle size but smaller than the lengthscale over which the density varies. We also average over a time $\Delta t$ that is longer than a few collision times but shorter than the timescale over which the density changes. The average $\bar{F}_{r \phi}$ is defined via

$$
(\Delta t \Delta r) \bar{F}_{r \phi} \equiv \int_{t}^{t+\Delta t} \int_{r}^{r+\Delta r} d t^{\prime} d r^{\prime}\left(n r^{\prime} v_{r} v_{\phi}\right)
$$

where $\boldsymbol{v}$ is a Lagrangian quantity that is tied to particles. Extracting $F_{r \phi}$ from a numerical simulation with only a spatial average $\left(\left\langle v_{r} v_{\phi}\right\rangle\right)$ can lead to large errors. In particular, in an optically thin disk, only a small fraction of the particles have collided within the last orbital period. Hence most particles contribute negligibly to $\left\langle v_{r} v_{\phi}\right\rangle$, and a calculation of $\left\langle v_{r} v_{\phi}\right\rangle$ can become plagued by small-number statistics. $\Delta t$,

For a single particle that does not collide in the interval

$$
\int_{t}^{t+\Delta t} v_{r} v_{\phi} d t^{\prime}=-\Omega^{-1} \int \frac{d v_{\phi}^{2}}{d t} d t=-\Omega^{-1}\left(\left.v_{\phi}^{2}\right|_{t+\Delta t}-\left.v_{\phi}^{2}\right|_{t}\right) .
$$

But if it collided once,

$$
\int_{t}^{t+\Delta t} v_{r} v_{\phi} d t^{\prime}=-\Omega^{-1}\left(\left.v_{\phi}^{2}\right|_{t+\Delta t}-\left.v_{\phi}^{2}\right|_{t}-\delta\left(v_{\phi}^{2}\right)\right)
$$

where $\delta$ represents the change due to the collision. Therefore each collision contributes to Equation (42) in the amount of

$$
(\Delta t \Delta r) \delta \bar{F}_{r \phi}=r \Omega^{-1}\left(\delta\left(v_{\phi, 1}^{2}\right)+\delta\left(v_{\phi, 2}^{2}\right)\right),
$$

where $\delta\left(v_{\phi, 1}^{2}\right), \delta\left(v_{\phi, 2}^{2}\right)$ are the contributions from the two collision partners. The contribution from the endpoints, $\left.v_{\phi}^{2}\right|_{t+\Delta t}-\left.v_{\phi}^{2}\right|_{t}$, can be neglected as long as $\Delta t$ is much longer than the collision time.

We define

$$
(\Delta t \Delta r) \bar{F}_{\mathrm{NL}} \equiv \iint d t^{\prime} d r^{\prime} F_{\mathrm{NL}}
$$

If two particles collide when their positions are at radii $r_{1}, r_{2}$ (where $r<r_{2}<r_{1}<r+\Delta r$ ), and if the particle at $r_{1}$ has its $\phi$-velocity changed by $\delta v_{\phi, 1}$ in the collision, then the collision contributes to the integral in Equation (46) in the amount of

$$
\begin{aligned}
(\Delta t \Delta r) \delta \bar{F}_{\mathrm{NL}} & =\left(r_{1}-r_{2}\right) r_{1} \delta v_{\phi, 1} \\
& \approx r\left(r_{1}-r_{2}\right)\left(\delta v_{\phi, 1}-\delta v_{\phi, 2}\right) / 2 .
\end{aligned}
$$

To obtain the latter symmetric form, we approximated $r_{1} \approx r$ and $\delta v_{\phi, 1} \approx-\delta v_{\phi, 2}$ (when in fact $r_{1} \delta v_{\phi, 1}=$ $\left.-r_{2} \delta v_{\phi, 2}\right)$. The error accrued is of order $\left(r_{1}-r_{2}\right) / r \sim$ $s / r \ll 1$, where $s$ is the particle size.
Since the number of collisions per unit time per unit radius is $n / 2 t_{\mathrm{col}}$,

$$
\begin{aligned}
& \bar{F}_{r \phi}=\frac{n}{2 t_{\text {col }}} \frac{2 r}{\Omega}\left\langle\delta\left(v_{\phi}^{2}\right)\right\rangle_{c}, \\
& \bar{F}_{\mathrm{NL}}=\frac{n}{2 t_{\mathrm{col}}} \frac{r}{2}\left\langle\left(r_{1}-r_{2}\right) \delta\left(v_{\phi, 1}-v_{\phi, 2}\right)\right\rangle_{c}
\end{aligned}
$$

where \langle\rangle$_{c}$ (not to be confused with \langle\rangle ) is an average over collisions, and $\left\langle\delta\left(v_{\phi}^{2}\right)\right\rangle_{c} \equiv\left\langle\delta\left(v_{\phi, 1}^{2}+v_{\phi, 2}^{2}\right)\right\rangle_{c} / 2$. With our collision algorithm, $t_{\text {col }}$ may be pulled out of the averages. We use Equations (49) and (50) to extract $\bar{F}_{r \phi}$ and $\bar{F}_{\text {NL }}$ from the simulations (see Figure 8 below).

We can estimate the magnitudes of the two fluxes as follows. Since the peculiar velocity distribution is anisotropic, with $\left\langle v_{\phi}^{2}\right\rangle=\left\langle v_{r}^{2}\right\rangle / 4<\left\langle v_{r}^{2}\right\rangle$ (Eqs. [40][41]), and since collisions tend to isotropize the distribution, therefore collisions systematically increase $v_{\phi}^{2}$ by $\left\langle\delta\left(v_{\phi}^{2}\right)\right\rangle_{c} \sim v^{2} \sim+e_{\mathrm{rms}}^{2}(r \Omega)^{2}$, transporting of $F_{r \phi}$ outwards. The contribution to $F_{\mathrm{NL}}$ is $\left\langle\left(r_{1}-r_{2}\right) \delta\left(v_{\phi, 1}-\right.\right.$ $\left.\left.v_{\phi, 2}\right)\right\rangle_{c} \sim+\Omega s^{2}$, where $s$ is the particle size. This is true even when $e_{\text {rms }} \gg s / r$ (i.e., when $\epsilon \rightarrow \epsilon_{*}$ ), because in that case $\delta\left(v_{\phi, 1}-v_{\phi, 2}\right)$ is nearly random and hence nearly uncorrelated with $r_{1}-r_{2}$. But because of the mean Keplerian shear, a small correlation $\sim \Omega s^{2}$ persists. This contribution also transports angular momentum outward. In sum,

$$
\begin{aligned}
& \bar{F}_{r \phi} \sim r \Omega \frac{n}{t_{\mathrm{col}}}\left(r e_{\mathrm{rms}}\right)^{2} \\
& \bar{F}_{\mathrm{NL}} \sim r \Omega \frac{n}{t_{\mathrm{col}}} s^{2} .
\end{aligned}
$$

\subsubsection{Relating $n \dot{\mathcal{E}}$ to $F_{H}^{\text {visc }}$}

We now calculate the energy lost in an inelastic collision, and thereby re-derive Equation (24) from a microscopic perspective. Consider the collision of two particles having total velocities $\boldsymbol{V}_{1,2}=\boldsymbol{v}_{1,2}+\boldsymbol{V}_{1,2}^{\text {circ }}$, where $\boldsymbol{V}_{1,2}^{\text {circ }}=\Omega\left(r_{1,2}\right) r_{1,2} \hat{\boldsymbol{\phi}}$ is the circular Keplerian speed at the positions of the particles. Then the specific energy lost per collision is, in previous notation,

$$
\begin{aligned}
(\Delta t \Delta r) \delta(\overline{n \dot{\mathcal{E}}})= & -\delta\left(V_{1}^{2}+V_{2}^{2}\right) / 2 \\
= & -\delta\left(v_{1}^{2}+v_{2}^{2}\right) / 2 \\
& -\delta \boldsymbol{v}_{1} \cdot \boldsymbol{V}_{1}^{\mathrm{circ}}-\delta \boldsymbol{v}_{2} \cdot \boldsymbol{V}_{2}^{\mathrm{circ}} \\
= & -\delta\left(v_{1}^{2}+v_{2}^{2}\right) / 2 \\
& -\left(r_{1}-r_{2}\right) r_{1} \delta v_{\phi, 1} d \Omega / d r \\
\approx & -\delta\left(v_{1}^{2}+v_{2}^{2}\right) / 2 \\
& -\left(r_{1}-r_{2}\right) r(d \Omega / d r) \times \\
& \left(\delta v_{\phi, 1}-\delta v_{\phi, 2}\right) / 2,
\end{aligned}
$$

where to derive (55) we used conservation of orbital angular momentum in a collision $\left(r_{1} \delta v_{\phi, 1}=-r_{2} \delta v_{\phi, 2}\right)$. Therefore

$$
\overline{n \dot{\mathcal{E}}}=-\frac{n}{2 t_{\mathrm{col}}}\left\langle\delta\left(v^{2}\right)+\frac{r}{2} \frac{d \Omega}{d r}\left(r_{1}-r_{2}\right) \delta\left(v_{\phi, 1}-v_{\phi, 2}\right)\right\rangle_{c} .
$$

We re-write the first term using $v^{2}=-3 v_{\phi}^{2}+e^{2}(r \Omega)^{2}$ (Eqs. [40]-[41]). Since the particles are in collisional equilibrium,

$$
\left\langle\delta\left(e^{2}\right)\right\rangle_{c}=0
$$

which implies that $\left\langle\delta\left(v^{2}\right)\right\rangle_{c}=-3\left\langle\delta\left(v_{\phi}^{2}\right)\right\rangle_{c}$. Inserting this into Equation (57), and comparing the result with Equations (49)-(50), completes our proof of Equation (24). 


\subsubsection{Calculating $F_{r \phi}$ and $\epsilon_{*}$}

We calculate the numerical constant that we dropped in Equation (51), and then use that result to calculate $\epsilon_{*}$. Goldreich \& Tremaine (1978) perform a similar calculation, with a different (though still idealized) collisional cross-section. Shukhman (1984) accounts for $F_{\mathrm{NL}}$ as well. The treatments of Goldreich \& Tremaine (1978) and Shukhman (1984) are more rigorous than ours, as they integrate over the velocity distribution function. They also consider the more general case of disks with order-unity optical depth. But their final expressions are "extremely cumbersome" (Shukhman 1984). Although our treatment is not rigorous, it is considerably simpler, and we justify it by comparing with simulations. We neglect $F_{\mathrm{NL}}$ in the present subsection, taking $e_{\mathrm{rms}} \gg s / r$.

For $\bar{F}_{r \phi}$ (Eq. [49]), we seek

$$
\left\langle\delta\left(v_{\phi}^{2}\right)\right\rangle_{c}=\left\langle v_{\phi}^{2}\right\rangle_{a c}-\left\langle v_{\phi}^{2}\right\rangle_{b c},
$$

where \langle\rangle$_{b c}$ is an average over collisions of the state immediately before the collision, and \langle\rangle$_{a c}$ is of the state immediately after. In an optically thin disk, the averages over particles are equal to time-averages for a single particle:

$$
\left\langle v_{r}^{2}\right\rangle=\frac{e_{\mathrm{rms}}^{2}}{2}(r \Omega)^{2}, \quad\left\langle v_{\phi}^{2}\right\rangle=\frac{e_{\mathrm{rms}}^{2}}{8}(r \Omega)^{2},
$$

using Equations (40)-(41). With our collision algorithm, the probability that a particle collides is uniform in time. Therefore

$$
\left\langle v_{\{r, \phi\}}^{2}\right\rangle_{b c}=\left\langle v_{\{r, \phi\}}^{2}\right\rangle .
$$

In collisional equilibrium (Eq. [58]),

$$
\left\langle\delta\left(v_{r}^{2}+4 v_{\phi}^{2}\right)\right\rangle_{c}=0 .
$$

We now evaluate $\left\langle v_{r}^{2}\right\rangle_{a c}$ and $\left\langle v_{\phi}^{2}\right\rangle_{a c}$. We make the plausible assumption that the relative velocity of collision partners, ${ }^{7}$ $\mathbf{u}=\mathbf{v}_{1}-\mathbf{v}_{2}$, is isotropically distributed after the collision:

$$
\left\langle u_{r}^{2}\right\rangle_{a c}=\left\langle u_{\phi}^{2}\right\rangle_{a c} .
$$

This assumption is verified by numerical simulation in §6.4. To relate $\mathbf{u}$ to $\mathbf{v}$,

$$
\begin{gathered}
\left\langle u_{\{r, \phi\}}^{2}\right\rangle_{b c}=2\left\langle v_{\{r, \phi\}}^{2}\right\rangle_{b c} \\
\left\langle\delta\left(u_{\{r, \phi\}}^{2}\right)\right\rangle_{c}=4\left\langle\delta\left(v_{\{r, \phi\}}^{2}\right)\right\rangle_{c} .
\end{gathered}
$$

For the first relation, we neglected the correlation between $\boldsymbol{v}_{1}$ and $\boldsymbol{v}_{2}$ before a collision, and for the second relation, we used $\delta\left(\boldsymbol{v}_{1}+\boldsymbol{v}_{2}\right)=0$. Equations (63)-(65) yield $\left\langle\delta\left(v_{\phi}^{2}-v_{r}^{2}\right)\right\rangle_{c}=(1 / 2)\left\langle v_{r}^{2}-v_{\phi}^{2}\right\rangle_{b c}$, which, with Equations (60)-(62) becomes

$$
\left\langle\delta\left(v_{\phi}^{2}\right)\right\rangle_{c}=\frac{3}{80} e_{\mathrm{rms}}^{2}(r \Omega)^{2},
$$

giving the numerical constant for $\bar{F}_{r \phi}$ (Eq. [49]).

To calculate $\epsilon_{*}$, we evaluate the specific energy lost per collision in the center-of-mass frame:

$$
-\frac{1}{4}\left\langle\delta\left(u^{2}\right)\right\rangle_{c}=\frac{9}{80} e_{\mathrm{rms}}^{2}(r \Omega)^{2},
$$

(Eqs [62], [65], [66]). We can also evaluate the energy loss as follows: from Equation (3), it is

$$
\frac{1}{4}\left(1-\epsilon^{2}\right)\left\langle u_{\mathrm{rel}, \|}^{2}\right\rangle_{b c}=\left(1-\epsilon^{2}\right) \frac{1}{8}\left\langle u^{2}\right\rangle_{b c},
$$

where we have made use of the isotropy of the pre-collision $\boldsymbol{u}$ with respect to the axis connecting the centers of the two particles. Since $\left\langle u^{2}\right\rangle_{b c}=(5 / 4) e_{\mathrm{rms}}^{2}(r \Omega)^{2}$, Equations (67) and (68) are equal only if $\epsilon$ is equal to

$$
\epsilon_{*}=\frac{\sqrt{7}}{5}=0.529 .
$$

This is interpreted as the one value for $\epsilon$ that enables the velocity distribution to equilibrate in the limit that $e_{\mathrm{rms}} \gg s / r$, i.e., $F_{r \phi} \gg F_{\mathrm{NL}}$.

\subsection{Dynamics from a Microscopic Perspective: Simulations}

In Figure 8 we test the theoretical results derived in §6.3. In the top panel, for the simulation with the same initial conditions as the one with $\epsilon=0.525$ in Figures 2 and 7 , we compute $\left\langle\delta\left(v_{\phi}\right)^{2}\right\rangle_{c}$ - the key factor that enters into $F_{r \phi}$ (Equation [49]) - in two ways: first as an average over all particles that collide within successive time intervals of $2000 \mathrm{yr}$, and second using Equation (66), where $e_{\mathrm{rms}}^{2}=\left\langle e^{2}\right\rangle$ is a spatial, not temporal, average over all particles (regardless of whether they collide). The agreement verifies Equation (66). Also shown in the top panel is the corresponding contribution to $F_{\mathrm{NL}}$, i.e., $(\Omega / 4)\left\langle\left(r_{1}-r_{2}\right) \delta\left(v_{\phi, 1}-v_{\phi, 2}\right)\right\rangle_{c}$ (Equation [50]). That this quantity is constant with time and sits far below $\left\langle\delta\left(v_{\phi}\right)^{2}\right\rangle_{c}$ is expected from Equations (51)-(52), given this simulation in which $e_{\mathrm{rms}} \gg s_{\text {grid }} / \bar{r}$ (i.e., $\epsilon_{*}-\epsilon \ll \epsilon_{*}$ ).

In the bottom panel of Figure 8, we test our assumption, made in Equation (63), that post-collision relative velocities are isotropic. The measured near-equality between $\left\langle u_{r}^{2}\right\rangle_{a c}$ and $\left\langle u_{\phi}^{2}\right\rangle_{a c}$ is satisfactory.

Finally, returning to Figure 7 , we plot separately the contributions to the total energy dissipation from $F_{r \phi}$ and $F_{\mathrm{NL}}$. The former contribution is described by

$$
k_{E}^{r \phi} \equiv \frac{-F_{r \phi}(d \Omega / d r)}{n^{2} s_{\text {grid }}^{4}\left(3 \Omega^{2} / 8 r t_{\text {orb }}\right)}
$$

(see Equations [24], [26], and [39]). We measure $F_{r \phi}$ according to

$$
\bar{F}_{r \phi} \doteq\left(\frac{r}{\Omega}\right) \frac{\delta\left(v_{\phi}^{2}\right)_{>r_{1}}-\delta\left(v_{\phi}^{2}\right)_{>r_{2}}}{d t_{m}\left(r_{1}-r_{2}\right)}
$$

where $\delta\left(v_{\phi}^{2}\right)_{>r}$ is the total change in $v_{\phi}^{2}$ summed over particles that collide between times $t_{m}$ and $t_{m}+d t_{m}$, at radii $>r$. For simplicity, we evaluate $k_{E}^{\mathrm{NL}}=k_{E}-k_{E}^{r \phi}$.

7 Since we take $e \gg s / r$, we neglect the difference in the Keplerian circular velocities at the positions of the two particles. 


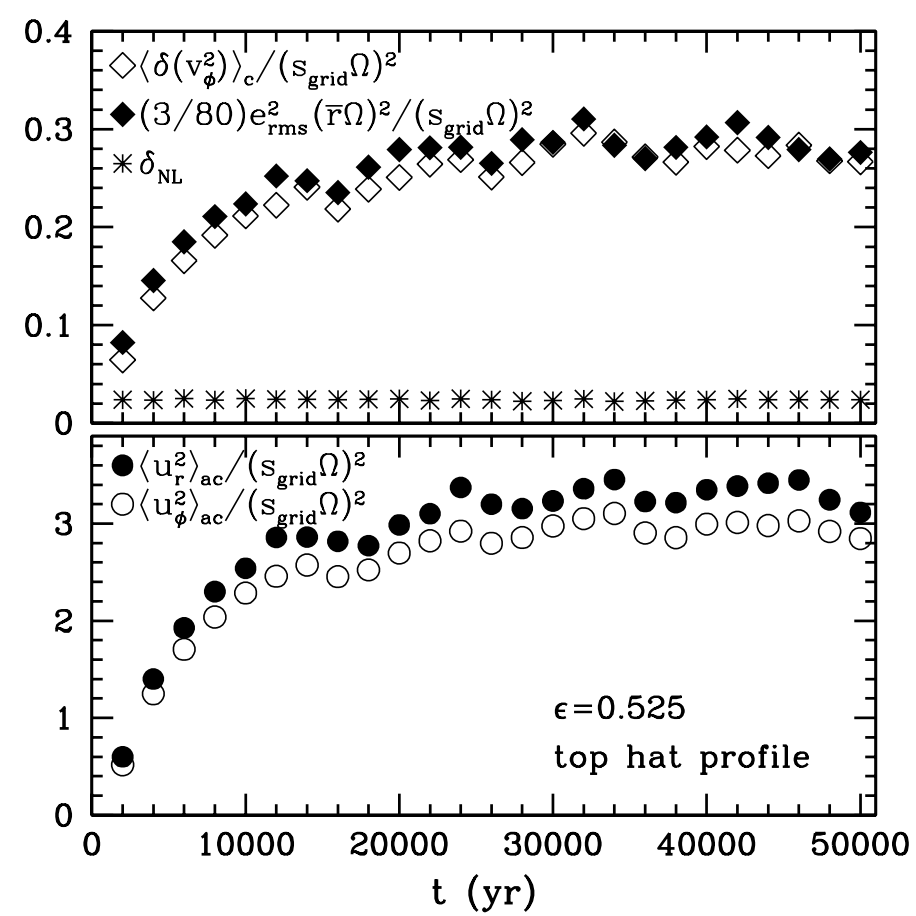

FIG. 8.- Averages over Collisions. The two panels show the time evolution of various collisional averages in a simulation with the same initial conditions as the one with $\epsilon=0.525$ in Figures 2 and 7 . Top panel: open diamonds show the factor that enters into $F_{r \phi}$ (Eq. [49]), i.e., $\left\langle\delta\left(v_{\phi}^{2}\right)\right\rangle_{c}=(1 / 2)\left\langle\delta\left(v_{\phi, 1}^{2}+v_{\phi, 2}^{2}\right)\right\rangle_{c}$, normalized as shown. The averaging over collisions is done by recording the peculiar velocities of colliding particles immediately before and after each collision. The averaging time is $2000 \mathrm{yr}$. Solid diamonds give $e_{\mathrm{rms}}^{2}=\left\langle e^{2}\right\rangle$ (a spatial, not temporal, average over all particles), multiplied by the appropriate pre-factor as given by Equation (66). The agreement between open and closed diamonds confirms Equation (66). Stars give the non-local contribution, appropriately normalized relative to the open diamonds, i.e., $\delta_{N L} \equiv(\Omega / 4)\left\langle\left(r_{1}-r_{2}\right) \delta\left(v_{\phi, 1}-v_{\phi, 2}\right)\right\rangle_{c} /\left(s_{\text {grid }} \Omega\right)^{2}$ (Eqs. [49]-[50]). Bottom panel: post-collision relative velocities are indeed nearly isotropic, as we had surmised in Equation (63). For this figure, we take $\boldsymbol{u}=\boldsymbol{V}_{1}-\boldsymbol{V}_{2}$, the total relative velocity; it includes the difference in the circular Keplerian velocities at the locations of the two particles.

\section{SUMMARY AND OUTLOOK}

We have introduced an algorithm to simulate collisions between inelastic particles in an optically thin disk orbiting a central mass. The algorithm is simple to implement and adds negligible running time to existing collisionless N-body codes. A major feature of the algorithm is that the disk particles' motions need only be tracked in a plane. Yet the algorithm transcends its two-dimensional appearance to simulate a three-dimensional disk of particles whose random velocity distribution tends to be isotropized by collisions.

We have performed a battery of tests of the algorithm for the case of an isolated, narrow, circular ring. Numerical simulations agree with analytic theory with regard to how the particles' velocity dispersion equilibrates, how the ring viscously spreads, how energy and angular momentum are transported, and how energy dissipation relates to the viscous angular momentum flux and to the background shear. Angular momentum transport arises not only from particle advection $\left(H F_{n}\right)$, but also from correlations in the random velocity field $\left(F_{r \phi}\right)$ and from finite particle sizes $\left(F_{\mathrm{NL}}\right)$. The relative magnitudes of each of these three terms can be measured from simulations. In making these and other measurements, we sought ways to minimize noise introduced by finite particle numbers (Poisson fluctuations). For example, when measuring viscous fluxes of angular momentum and energy, it proves useful to consider only those particles that actually collide during the measurement interval.

The stage is now set for simulating more complicated systems - narrow eccentric rings (like the Maxwell and Titan ringlets of Saturn, or the Epsilon ring of Uranus), and circumstellar disks with embedded planets. Among the phenomena we are interested in exploring numerically are the formation of sharp edges by shepherd satellites, the evolution of narrow rings into states of rigid apsidal precession, and the eccentricity evolution of planets as driven by disks.

We thank Ruth Murray-Clay for helpful exploratory calculations, and Jack Wisdom for encouraging remarks. EC acknowledges support from the National Science Foundation, NASA, and the Alfred P. Sloan Foundation, and is grateful for the warm hospitality of the Canadian Institute for Theoretical Astrophysics / University of Toronto, where a portion of this work was completed. 


\section{REFERENCES}

Brahic, A. 1977, A\&A, 54, 895

Charnoz, S., Thébault, P., \& Brahic, A. 2001, A\&A, 373, 683

Chiang, E. I. \& Culter, C. J. 2004, ApJ, 599, 675

Goldreich, P., Lithwick, Y., \& Sari, R. 2004, ARA\&A, 42, 549

Goldreich, P. \& Sari, R. 2003, ApJ, 585, 1024

Goldreich, P. \& Tremaine, S. D. 1978, Icarus, 34, 227

Greenberg, R. 1988, Icarus, 75, 527

Levison, H. F. \& Duncan, M. J. 1994, Icarus, 108, 18

Lewis, M. C. \& Stewart, G. R. 2000, AJ, 120, 3295
Lynden-Bell, D. \& Pringle, J. E. 1974, MNRAS, 168, 603

Petit, J.-M. \& Henon, M. 1987, A\&A, 188, 198

Press, W. H., Teukolsky, S. A., Vetterling, W. T., \& Flannery, B. P. 1992, Numerical recipes in FORTRAN. The art of scientific computing (Cambridge: University Press, —c1992, 2nd ed.)

Shukhman, I. G. 1984, Soviet Astronomy, 28, 574

Trulsen, J. 1971, Ap\&SS, 12, 329

Wisdom, J. \& Holman, M. 1991, AJ, 102, 1528

Wisdom, J. \& Tremaine, S. 1988, AJ, 95, 925 